\title{
Immediate Reproducibility of Clinical and Nonclinical Forms of Induced Ventricular Tachycardia
}

\author{
MICHAEL de BUITLEIR, MD, FRED MORADY, MD, LORENZO A. DiCARLO, Jr., MD, \\ JEFFREY M. BAERMAN, MD, and RYSZARD B. KROL, MD
}

This prospective study assessed the immediate reproducibility of clinical and nonclinical forms of ventricular tachycardia (VT) induced by programmed ventricular stimulation. Twenty-three clinical VTs were unimorphic and previously documented and 22 nonclinical VTs (17 polymorphic and 5 unimorphic) were induced in patients with either no documented or suspected history of VT, or documented VT that had a configuration different from that of the induced VT. The stimulation protocol included 1 to 3 ventricular extrastimuli, 2 drive cycle lengths, and 2 right ventricular stimulation sites. Each VT was induced on the first attempt, then the stimulation protocol was repeated twice in the drug-free state. After the first VT induction, 21 of 23 clinical VTs $(91 \%)$ and 17 of 22 nonclinical VTs $(77 \%)$ were reinduced on the second attempt. After 2 VT inductions, 21 of 21 clinical VTs $(100 \%)$ and 15 of 17 nonclinical VTs $(88 \%)$ were reinduced on the third attempt. The reinduction rates of the clinical and nonclinical VTs were not significantly different. Among the clinical VTs, the reproducibility of the induction technique was $81 \%$ after 1 induction and $88 \%$ after 2 inductions with the same technique. These results imply that (1) acute drug testing can be reliably performed after 2 inductions but not 1 induction of clinical VT; (2) reproducibility is not helpful in determining whether an induced VT is clinical or nonclinical; and (3) changes in induction technique during drug testing should be interpreted with caution because changes may occur in the absence of drugs.
$\mathbf{R}$ N eliable assessment of drug efficacy during acute electropharmacologic testing depends on the immediate reproducibility of ventricular tachycardia (VT) induced by programmed ventricular stimulation. It has been suggested that an increase in the number of extrastimuli required to induce VT after administration of a drug may predict a good clinical response to that drug. ${ }^{1,2}$ However, no studies have critically analyzed the immediate reproducibility of either VT induction or the number of extrastimuli required to induce VT.

Programmed ventricular stimulation may induce not only clinical forms of VT, but also nonclinical forms. ${ }^{3,4}$ In a patient with suspected but undocumented VT, it may be unclear whether the VT induced by programmed ventricular stimulation is clinically im-

From the Cardiology Division, Department of Internal Medicine, University of Michigan Medical Center, Ann Arbor, Michigan. Manuscript received December 12, 1985; revised manuscript received and accepted March 6, 1986.

Address for reprints: Fred Morady, MD, Division of Cardiology, F245C, University Hospital, 1500 East Medical Center Drive, Ann Arbor, Michigan 48109-0366. portant. It has been suggested that the ability to reproducibly induce a particular form of VT implies that the VT is clinically significant. ${ }^{5}$ Howcver, no studics have compared the immediate reproducibility of clinical and nonclinical forms of VT.

This prospective study was designed to answer 3 questions: If a particular form of VT is inducible on 2 attempts, does this guarantee inducibility on a third attempt? When VT can be induced on 3 attempts, what is the reproducibility of the induction technique that induced VT on the first attempt? Is there a difference in reproducibility of clinical and nonclinical forms of VT induced by programmed ventricular stimulation?

\section{Methods}

Study participants consisted of 42 consecutive patients undergoing electrophysiologic study and meeting the entry criteria: VT was inducible by programmed ventricular stimulation, VT did not require direct-current countershock to terminate, and the patient had either a history of sustained unimorphic VT documented on a 12-lead electrocardiogram or no history of documented or suspected VT. Each patient 
with no history of VT had at least one 24-hour ambulatory electrocardiographic recording demonstrating absence of more than 3 consecutive ventricular premature complexes. Induced VT was categorized as clinical when it was identical to the patient's spontaneous VT, as documented by a 12-lead electrocardiographic recording. Induced VT was categorized as nonclinical if it was induced in patients with no history of documented or suspected VT or if it was induced in a patient with documented VT but had a different configuration than the documented VT. Sustained VT was defined as VT lasting at least 30 seconds. Nonsustained VT was defined as VT 6 beals to 30 seconds in duration.

Patient characteristics: Patient characteristics are described in Table I. In 3 patients, both clinical and nonclinical forms of VT were induced.

Characteristics of induced ventricular tachycardia: Twenty-three VTs were clinical and 22 were nonclinical. VT characteristics are listed in Table II.

The 23 clinical VTs were induced in patients who had a documented history of sustained unimorphic VT and who were undergoing electropharmacologic testing. Of the 22 nonclinical VTs, the 17 that were polymorphic and nonsustained were induced in patients

\section{TABLE I Patient Characteristics}

\begin{tabular}{lcc}
\hline & Clinical VT & Nonclinical VT \\
\hline No. of pts & $23^{*}$ & 19 \\
Males/females & $19 / 4$ & $16 / 3$ \\
Age (yr) (mean \pm SD) & $62 \pm 10$ & $55 \pm 14$ \\
Heart disease & & \\
Coronary artery disease & 19 & 11 \\
Dilated cardiomyopathy & 1 & 5 \\
Mitral valve prolapse & 1 & 0 \\
None & 2 & 3 \\
\hline
\end{tabular}

* Three patients also had inducible nonclinical VT.

$\mathrm{SD}=$ standard deviation; VT = ventricular tachycardia.

TABLE II Characteristics of Induced Ventricular Tachycardias

\begin{tabular}{lcc}
\hline & Clinical VTs & Nonclinical VTs \\
\hline No. of pts & 23 & 22 \\
Configuration & & \\
$\quad$ Right bundle branch block & 14 & 3 \\
$\quad$ Left bundle branch block & 9 & 2 \\
$\quad$ Polymorphic & 0 & 17 \\
Duration & & \\
$\quad$ Nonsustained ( 6 beats-30 sec) & 1 & 22 \\
$\quad$ Sustained $>30$ sec) & 22 & 0 \\
Cycle length (ms) (mean \pm SD) & $312 \pm 60$ & $212 \pm 56^{*}$ \\
No. of extrastimuli required to & & \\
$\quad$ induce VT on first attempt & 5 & 0 \\
1 & 13 & 11 \\
2 & 5 & 11 \\
$3 \quad$ & 21 & 16 \\
Right ventricular induction site & 2 & 6 \\
$\quad$ Apex & & \\
Outflow tract or septum & &
\end{tabular}

${ }^{*} p<0.001$ vs clinical VTs.

$\mathrm{SD}=$ standard deviation; $\mathrm{VT}=$ ventricular tachycardia with sick sinus syndrome, atrioventricular conduction disturbances, carotid hypersensitivity, paroxysmal supraventricular tachycardia or unexplained syncope undergoing programmed ventricular stimulation in the course of a complete electrophysiologic study. Nonsustained polymorphic VT induced in patients with unexplained syncope has been shown not to be of clinical significance. $4,6,7$ The 5 nonclinical VTs that were unimorphic were induced in patients with a history of sustained unimorphic VT, which had a configuration different from that of the induced VT.

Programmed ventricular stimulation protocol: Patients were studied in the fasting, unsedated state after they gave informed consent. Antiarrhythmic drug therapy was discontinued at least 4 half-lives before electrophysiologic study. Two No. $6 \mathrm{Fr}$ quadripolar electrode catheters were inserted percutaneously into a femoral vein and positioned against the right ventricular apex and either the right ventricular outflow tract or septum. A programmable stimulator (Bloom Associates, Ltd.) was used to deliver stimuli at twice diastolic threshold and $2 \mathrm{~ms}$ in duration. Leads $\mathrm{V}_{1}$, I and III and intracardiac electrograms were displayed on an oscilloscope and recorded on an Electronics for Medicine VR-16 recorder at a paper speed of $25 \mathrm{~mm} / \mathrm{s}$. When unimorphic VT was induced, a 12lead electrocardiogram was recorded.

Programmed ventricular stimulation was performed with single and double extrastimuli using 6- to 8 -beat drive trains, 2 drive cycle lengths $(600$ or $500 \mathrm{~ms}$ and $400 \mathrm{~ms}$ ), and $10-\mathrm{ms}$ decrements, first at the apex and then at the second right ventricular site. If an endpoint of the stimulation protocol was not reached, triple extrastimuli were then introduced, first at the apex and then at the second right ventricular site.

In patients who had a history of documented unimorphic VT, the endpoint of the stimulation protocol was induction of clinical VT. In patients with no history of documented or suspected VT, the endpoint was induction of any form of VT. If VT or ventricular fibrillation requiring direct-current countershock was induced, the patient was excluded from the study.

In each patient, VT was induced on the first attempt at programmed stimulation. The programmed ventricular stimulation protocol was immediately repeated twice after the first induction of VT. On the second and third attempts, programmed ventricular stimulation was initiated at the same site and basic drive cycle length used to induce VT on the first attempt. If VT was not induced, the remainder of the stimulation protocol was then carried out.

Statistical analysis was performed with a Student $t$ test and chi-square analysis. A p value $<0.05$ was considered statistically significant.

\section{Results}

Reproducibility after one induction of ventricular tachycardia: After 1 induction of VT, 21 of 23 clinical VTs $(91 \%)$ were induced on the second attempt. In 2 patients, both of whom had coronary artery disease, no VT was induced when the entire programmed ventricular stimulation protocol was repeated. One of the 
nonreproducible VTs was induced by double extrastimuli and 1 by triple extrastimuli on the initial attempt.

After 1 induction of VT, 17 of 22 nonclinical VTs $(77 \%)$ were induced on the second attempt. In 5 patients who originally had inducible nonclinical VT, no VT was induced when the entire stimulation protocol was repeated; 4 of these patients had coronary artery disease and 1 had dilated cardiomyopathy.

The $77 \%$ reinduction rate for the nonclinical VTs was not significantly different from the $91 \%$ reinduction rate for the clinical VTs.

Reproducibility after two inductions of ventricular tachycardia: Among the 21 clinical VTs that were induced twice, all 21 were again inducible on the third attempt.

Among the 17 nonclinical VTs that were induced twice, $15[88 \%]$ were inducible on the third attempt. This $88 \%$ reinduction rate for nonclinical VTs was not significantly different from the $100 \%$ reinduction rate for clinical VTs.

Reproducibility of induction technique: Among the 21 clinical VTs that were reproducibly induced on the first 2 attempts, 17 of $21(81 \%)$ were induced by the same number of extrastimuli on both occasions. One clinical VT required fewer extrastimuli to induce the second time, and 3 required more extrastimuli. All VTs were reinduced at the original stimulation site. Among 17 clinical VTs induced on 3 occasions that were induced by the same technique on the first 2 attempts, the same technique induced VT on the third attempt in 15 of 17 cases $(88 \%)$. One clinical VT required fewer extrastimuli to induce the third time and 1 required more extrastimuli.

Among the 17 nonclinical VTs that were reproducibly induced on the first 2 attempts, 16 of 17 (94\%) were induced by the same number of extrastimuli on both attempts. Among 14 nonclinical VTs induced on 3 occasions that were induced by the same technique on the first 2 attempts, the same technique induced VT on the third attempt in 12 of 14 cases (86\%).

\section{Discussion}

No studies to date have addressed the question of how many times a clinical VT must be induced in the control state before the results of acute electropharmacologic testing can be meaningfully interpreted. The rcsults of the present study indicate that acute electropharmacologic testing can be reliably performed after 2 inductions of VT, but not after 1 . Approximately $10 \%$ of clinical VTs that were induced once were not inducible on a second attempt at programmed ventricular stimulation. However, clinical VTs induced twice were always inducible a third time. Therefore, 2 inductions of a given form of clinical VT in the control state appear adequate to ensure that the absence of inducible VT during acute drug testing is due to a drug effect and not random noninducibility.

Although 2 inductions of a clinical VT predicted a high degree of reproducibility in inducing the VT on a third occasion, the reproducibility of the specific induction technique was not as high. Even when VT is induced by the same step in the stimulation protocol on 2 consecutive occasions, there is approximately a $10 \%$ chance that VT induction will require fewer or more extrastimuli on the third attempt.

This observation has 2 important implications regarding electropharmacologic testing. First, because even in the control state induction of VT on a third attempt may require more extrastimuli than requircd on the first 2 attempts, during drug testing it may not be adequate to stimulate only up to the step in the protocol that induced VT in the control state; even if VT was induced on 2 occasions in the control state with 1 or 2 extrastimuli, the drug testing protocol should include 3 extrastimuli to ensure that an apparent drug response is not due to random change in the induction technique. Second, because the induction technique may change in the absence of drugs, a change in the number of extrastimuli required to induce VT during drug testing must be interpreted with caution. A decrease in the number of extrastimuli required to induce VT during drug testing has been suggested as indicating a proarrhythmic effect. ${ }^{8}$ Furthermore, an increase in the number of extrastimuli required to induce VT during drug testing has been suggested as indicating a beneficial drug effect. ${ }^{1,2}$ However, the results of the present study suggest that after 2 inductions of VT by the same technique in the control state, there is at least a $10 \%$ chance that a change in the number of extrastimuli required to induce VT during drug testing is a random change not due to the drug. If VT is induced only once in the control state, this probability increases to approximately $20 \%$.

In patients in whom documentation of the spontaneous VT is adequate, VT induced by programmed ventricular stimulation can be clearly identified as being clinical or nonclinical. However, in patients with inadequately documented VT or suspected but undocumented VT, the clinical significance of an induced VT may be unclear. It has been suggested that the ability to reproducibly induce a particular form of VT may indicate that the VT is clinically significant. ${ }^{5}$ However, the results of the present study demonstrate that reproducibility of an induced VT does not distinguish clinical from nonclinical tachycardia. Although reproducibility of nonclinical VTs tended to be lower than that of clinical VTs, there was no significant difference between the 2 . Therefore, the ability to reproducibly induce a VT of unclear significance should not be considered evidence that the VT is clinical.

All of the clinical VTs in this study were unimorphic. Although sustained polymorphic VT may, at times, be a clinical arrhythmia, as in patients who have had an out-of-hospital cardiac arrest, the reproducibility of this type of VT was not assessed in this study for 2 rcasons: repeated induction of sustained polymorphic VT may have necessitated multiple direct-current countershocks, and even in patients with out-of-hospital cardiac arrest, the clinical significance of sustained polymorphic VT may be unclear. ${ }^{4}$ Therefore, the conclusions of this study regarding reproducibility of clinical VTs are applicable only to sustained unimorphic VT. 
The nonclinical VTs in this study included both nonsustained polymorphic and sustained unimorphic tachycardias. The polymorphic VTs were considered nonclinical because they were induced in patients with no documented history of VT or in patients with a history of only unimorphic VT. The nonclinical unimorphic VTs were induced in patients with a history of documented unimorphic VT. Patients with a documented history of unimorphic VT often have multiple morphologic patterns of unimorphic VT and an induced unimorphic VT should therefore not be categorized as nonclinical unless every spontaneous episode has been documented. ${ }^{9}$ This was the case in the patients in this study.

In conclusion, inability to induce VT during acute electropharmacologic testing can be considered a valid response if the VT was induced at least twice before the drug was administered. However, the induction technique may be variable even in the absence of drugs; therefore, the apparent effects of a drug on the number of extrastimuli required to induce VT must be interpreted with caution. Finally, reproducibility of an induced VT is not a valid criterion by which the VT can be judged to be clinical or nonclinical.

Acknowledgment: We thank Lisa Hackbarth for her excellent secretarial assistance, the nurses and technicians of the Clinical Electrophysiology Labora- tory for assistance in performing the electrophysiology studies, and Stephen Schmaltz for computer and statistical assistance.

\section{References}

1. Breithardt G, Borggrefe M, Seipel L. Selection of optimal drug treatment of ventricular tachycardia by programmed electrical stimulation of the heart. Ann NY Acad Sci 1984:427:49-66.

2. Naccarelli GV, Fineberg NS, Zipes DP, Heger JI, Duncan G, Prystowsky EN. Amiodarone: risk factors for recurrence of symptomatic ventricular tachycardia identified at electrophysiologic study. IACC 1985;6:814-812.

3. Brugada P, Wellens HJJ. Programmed electrical stimulation of the heart in ventricular arrhythmias. Am I Cardial 1985;56:187-190.

4. Wellens HJ, Brugada P, Stevenson WG. Programmed electrical stimulation of the heart in patients with life-threatening ventricular arrhythmias: what is the significance of induced arrhythmias and what is the correct stimulution prolucol? Circulation 1985;72:1-7.

5. Schoenfeld MH, McGovern B, Garan H, Kelly E, Grant G, Ruskin IN. Determinants of the outcome of electrophysiologic study in patients with ventricular tachyarrhythmias. JACC 1985;6:298-306.

6. Morady F, Shen E, Schwartz A, Hess D, Bhandari A, Sung RJ, Scheinman MM. Long-term follow-up of patients with recurrent unexplained syncope evaluated by electrophysiologic testing, JACC 1983:2:1053-1059.

7. Doherty JU, Pembrook-Rogers D, Grogan EW, Falcone RA, Buxton AE, Marchlinksi FE, Gassidy DM, Kienzle MG, Almendral JM, Josephson ME. Electrophysiologic evaluation and follow-up characteristics of patients with recurrent unexplained syncope and presyncope. Am I Cardiol 1985;55:703708.

8. Poser RF, Podrid RI, Lombardi F, Lown B. Aggravation of arrhythmia induced with antiarrhythmic drugs during electrophysiologic testing. Am Heart I 1985;110:9-16.

9. Morady F, DiCarlo L, Winston S, Davis JC, Scheinman MM. A prospective comparison of triple extrastimuli and left ventricular stimulation in studies of ventricular tachycardia induction. Circulation 1984;70:52-57. 